\title{
Identifying Learning Styles in Learning Management Systems by Using Indications from Students' Behaviour
}

\author{
Sabine Graf* \\ National Central University \\ Graduate Institute of Learning \\ and Instruction, Taiwan \\ sabine.graf@ieee.org
}

\author{
Kinshuk \\ Athabasca University \\ School of Computing and \\ Information Systems, Canada \\ kinshuk@ieee.org
}

\author{
Tzu-Chien Liu \\ National Central University \\ Graduate Institute of Learning \\ and Instruction, Taiwan \\ ltc@cc.ncu.edu.tw
}

\begin{abstract}
Making students aware of their learning styles and presenting them with learning material that incorporates their individual learning styles has potential to make learning easier for students and increase their learning progress. This paper proposes an automatic approach for identifying learning styles with respect to the Felder-Silverman learning style model by inferring their learning styles from their behaviour during they are learning in an online course. The approach was developed for learning management systems, which are commonly used in elearning. In order to evaluate the proposed approach, a study with 127 students was performed, comparing the results of the automatic approach with those of a learning style questionnaire. The evaluation yielded good results and demonstrated that the proposed approach is suitable for identifying learning styles. By using the proposed approach, students' learning styles can be identified automatically and be used for supporting students by considering their individual learning styles.
\end{abstract}

\section{Introduction}

The field of learning styles is complex and affected by several aspects, leading to different concepts and views. Many learning style models exist in literature, such as the learning style model by Kolb [1], Honey and Mumford [2], and Felder and Silverman [3]. While there are still many open issues with respect to learning styles, all learning style models agree that learners have different ways in which they prefer to learn. Furthermore, many educational theorists and researchers consider learning styles as an important

\footnotetext{
* The author would like to thank the National Science Council of the Republic of China, Taiwan, for financially supporting this research under Contract No. NSC 097-2811-S-008-001-.
}

factor in the learning process and agree that incorporating them in education has potential to facilitate learning for students.

Learning styles can be considered in different ways. A first step is to make learners aware of their learning styles and show them their individual strengths and weaknesses. The knowledge about their learning styles helps students to understand why learning is sometimes difficult for them and is the basis for developing their weaknesses.

Furthermore, students can be supported by matching the teaching style with their learning style. Providing students with learning material and activities that fit their preferred ways of learning can make learning easier for them. This matching hypothesis is supported by educational theories. Moreover, studies such as those by Bajraktarevic, Hall, and Fullick [4] and Graf and Kinshuk [5] demonstrated supportive results.

For considering learning styles in education, the students' learning styles need to be known first. Brusilovsky [6] distinguished between two different ways of student modelling: collaborative and automatic. In the collaborative approach, the learners provide explicit feedback which can be used to build and update a student model, such as filling out a learning style questionnaire. In the automatic approach, the process of building and updating the student model is done automatically based on the behaviour and actions of learners while they are using the system for learning. The automatic approach is direct and free from the problem of inaccurate self-conceptions of students. Moreover, it allows students to focus only on learning rather than additionally providing explicit feedback about their preferences. In contrast to learning style questionnaires, an automatic approach can also be more accurate and less error-prone since it analyses data from a specific time span rather than data which are gathered at one specific point of time. 
In this paper, we propose an automatic student modelling approach for identifying learning styles in learning management systems (LMSs). LMSs such as Moodle [7] and WebCT [8] are commonly and successfully used in e-learning. They aim at supporting teachers in creating and managing online courses and provide them with a great variety of features which can be included in the course such as learning material, quizzes, discussion forums, assignments, and so on. The proposed student modelling approach is developed in a generic way, based on commonly used features in LMSs, and is therefore applicable for LMSs in general.

Regarding the learning style model, we selected the Felder-Silverman learning styles model (FSLSM) [3]. FSLSM describes learning styles in very much detail by characterising each learner according to four dimensions: active/reflective, sensing/intuitive, visual/ verbal, and sequential/global.

In the following section, the concept for identifying learning styles is introduced. Subsequently, the evaluation of the proposed approach and its results are presented. Section 4 provides discussion about the proposed approach and introduces related works. Section 5 concludes the paper.

\section{A concept for identifying learning styles}

In the following subsections, the investigated patterns of behaviour for each learning style dimension as well as the concept for calculating learning styles from these patterns are presented.

\subsection{Relevant patterns of behaviour}

In order to make our approach applicable for LMSs in general, only commonly used features in LMSs were selected to be the basis for patterns. These features include: content objects, outlines, examples, selfassessment tests, exercises, and discussion forums. Furthermore, the navigation behaviour of students in the course was considered.

In the next subsections, the characteristics of each learning style with respect to FSLSM are described and the relevant patterns for identifying learning styles for each dimension are presented, using the literature regarding FSLSM [3] as basis.

2.1.1. Active/reflective dimension. Active learners are characterised as learners who prefer to process information actively by doing something with the learned material, for example discussing, explaining, or testing it. On the other hand, reflective learners prefer to think about the material and work alone. Regarding discussing and explaining, communication tools like discussion forums can give indications about the students' preference for active or reflective learning. While active learners are expected to post more often in order to ask, discuss, and explain something, reflective learners are supposed to prefer to participate passively by carefully and frequently reading the postings but only rarely posting by themselves. Due to the preference of testing and trying things out, active learners are expected to perform more self-assessment tests and more exercises as well as spend overall more time on exercises. Furthermore, they are supposed to spend only little time on examples since they prefer doing something by themselves rather than looking at how someone else has solved a problem. Since reflective learners like to think and reflect about the material, they are expected to visit and spend more time on reading material like content objects as well as stay longer at outlines. They also tend to take longer on self-assessment tests as well as on the result pages of self-assessments and exercises for reflecting on their results. As a consequence, reflective learners are also expected to answer the same question in a selfassessment test less often twice wrong.

2.1.2. Sensing/intuitive dimension. Since sensing learners favour concrete material like facts and data, whereas intuitive learners prefer to learn abstract material such as theories and their underlying meaning, analysing the performance on questions about facts as well as on theories and concepts provides an indication about the preferred learning style. Furthermore, in order to learn from concrete material, sensing learners tend to prefer examples. Therefore, the visits and time spent on examples serve as other patterns. On the other hand, intuitive learners are supposed to learn from content objects and use examples only as supplementary material. Therefore, the number and time spent on content objects tend to be higher and the number and time spent on examples tend to be lower. Furthermore, sensing learners like to solve problems based on standard procedures, which can be again indicated by a high interest in examples in order to see and learn existing approaches and a high number of conducted self-assessment tests and exercises in order to check the acquired knowledge. On the other hand, intuitive learners tend to be more creative and like challenges. Therefore, they are expected to be better in answering questions about developing new solutions, which requires the understanding of underlying theories and concepts. Another characteristic of sensing learners is that they are more patient with details and work carefully but slowly. With respect to the preference for working slowly, the time taken for self-assessment tests is considered as pattern. Because these students tend to check their answers carefully 
before submitting, another pattern is the number of revisions performed before handing in a test or exercise. Another pattern is the time students spent on reviewing their results, where sensing learners again are expected to spend more time. Furthermore, their preference for being careful with details can be indicated by their performance on questions about details.

2.1.3. Visual/verbal dimension. While visual learners learn best from what they can see such as graphics, images, and flow charts, verbal learners prefer to learn from words, regardless whether they are spoken or written. Therefore, the performance on questions about graphics as well as on text can act as other patterns. Furthermore, verbal learners tend to like communicating and discussing with others. Thus, a high number of visits and postings as well as a high amount of time spent in a discussion forum can indicate a verbal learning style. Furthermore, verbal learners are expected to visit reading material such as content objects more often.

2.1.4. Sequential/global dimension. Sequential learners are more comfortable with details, whereas global learners tend to be good in seeing the "big picture" and connections to other fields. Therefore, the performance of questions dealing with overviews of concepts or connections between concepts and questions about details serve as patterns for this dimension. Because global learners are interested in getting the "big picture", outlines of the course and the chapters are especially important for them. A high number of visits and more time spent on such chapter outlines as well as on the course overview page indicate a global learning style. Furthermore, their interest in relating and connecting topics to each other helps them to interpret predefined solutions and develop new solutions. Therefore, they are expected to perform better on respective questions. The navigation of learners in a course acts also as a pattern denoting a sequential or global learning style. While sequential learners tend to go through the course step by step in a linear way, global learners tend to learn in large leaps, sometimes skipping learning objects and jumping to more complex material. Therefore, the number of skipped learning objects can act as a pattern.

\subsection{From behaviour to learning styles}

The previous section described the patterns which are incorporated for each dimension as well as whether a high or low occurrence indicates a specific learning style preference. Based on this information, data about students' behaviour can be used to calculate hints for specific learning style preferences. For example, if a learner often visited exercises, this gives us a hint that the learner prefers an active learning style. Hints are stated by four values: 3 indicates that the student's behaviour gives a strong indication for the respective learning style, 2 indicates that the student's behaviour is average and therefore does not provide a specific hint, 1 indicates that the student's behaviour is in disagreement with the respective learning style, and 0 indicates that no information about the student's behaviour is available. In order to classify the behaviour of students into these four groups, thresholds from literature are used as basis, considering additionally the characteristics of the respective course.

By summing up all hints and dividing them by the number of patterns that include available information, a measure for the respective learning style is calculated. This measure is then normalised on a range from 0 to 1 , where 1 represents a strong positive preference and 0 represents a strong negative preference for the respective learning style. If no pattern includes available information, no conclusion can be drawn.

\section{Evaluation}

The proposed student modelling approach was evaluated by a course about object oriented modelling, held at a university in Austria. 127 students participated in the study. The course included all types of learning objects described in the previous section. The LMS Moodle [7] was used and few extensions of its tracking mechanism were performed, allowing the system to gather data regarding all introduced patterns.

In order to classify the occurrence of behaviour with respect to the investigated patterns, thresholds were used for each pattern. In the next subsection, these thresholds are discussed. Subsequently, the evaluation method and the results of the evaluation are described.

\subsection{Classifying the occurrence of behaviour}

The thresholds for distinguishing whether students have a high, moderate, or low occurrence of behaviour regarding each pattern were determined from literature and adjusted based on the characteristics of the course rather than depending on the students' average behaviour in the class. Thus, the approach is also applicable for small classes, where the average distribution of learning style preferences might not apply due to its small size.

The thresholds regarding discussion forum were based on recommendations from Rovai and Barnum [9], but were lowered since the forum was mainly used 
for asking questions which were then answered by the tutors rather than discussing with other students. Therefore, for the number of visits, thresholds of 7 and 14 visits per week were used, for the time students spent on the forum, thresholds of 5 and 10 minutes per week were used, and for the number of postings, thresholds of 2 and 4 postings per course were used.

Based on the assumptions of García et al. [10], the thresholds for visiting exercises were set to $25 \%$ and $75 \%$ of available exercises. For self-assessment tests and examples, we used a threshold of 50\% and $100 \%$ since both types of learning objects were designed in a way that each object might be visited more than once. For outlines, thresholds of $75 \%$ and $150 \%$ were used. Regarding content objects, students had additionally the possibility to download the learning material for print. Therefore, the content objects were mainly used for looking up information when students were conducting, for example, some exercises or were reflecting about a topic. Therefore, the thresholds for visiting content objects were set to $10 \%$ and $20 \%$ of all available content objects. Furthermore, the thresholds for visiting the course overview page was determined with $10 \%$ and $20 \%$ of all visited learning objects.

The thresholds for the time spent on examples, exercises, self-assessment tests, content objects, outlines, and the course overview page were determined as $50 \%$ and $75 \%$ in relation to the expected learning time of students with high interest in the respective type of learning object, following the recommendation of García et al. [10].

For the time spent on the results of an exercise or self-assessment test, thresholds of 30 seconds and 60 seconds were assumed. Thresholds for the performance of specific question types were assumed as 50\% and $75 \%$ of correctly answered questions, based on the applied grading system. With respect to revisions of self-assessment tests and exercises, thresholds were determined as $2.5 \%$ and $5 \%$ of performed selfassessment tests or exercises. The thresholds regarding how often students answered a self-assessment question twice wrong were assumed as $25 \%$ and $50 \%$ of times a student is asked the same question twice.

Regarding skipping learning objects, we looked at how often students skipped learning objects in relation to the total number of visited learning objects. Thresholds of $1 \%$ and $2 \%$ of times students used the navigation menu to skip learning objects were assumed.

\subsection{Method of evaluation}

In order to evaluate our approach, its results were compared with the results of the Index of Learning Styles (ILS) questionnaire, a 44-item questionnaire which was developed by Felder and Soloman [11] for identifying learning styles based on the FSLSM. The proposed approach aims at detecting learning styles for each dimension of the FSLSM on a 3-item scale, distinguishing, for example, between an active, balanced, and reflective learning style. Therefore, the measure introduced in Section 2.2 was divided into three groups using values of 0.25 and 0.75 as thresholds. Similarly, results of the ILS questionnaire were divided into three groups. For measuring the precision of the proposed approach, including also how close the predicted learning style is to the learning style based on the ILS questionnaire, the following measure proposed by García et al. [10] was used:

$$
\text { Precision }=\frac{\sum_{i=1}^{n} \operatorname{Sim}\left(L S_{\text {predicted }}, L S_{I L S}\right)}{n} \cdot 100,
$$

where $L S_{\text {predicted }}$ refers to the learning style predicted by the proposed approach, using a 3-item scale, $L S_{I L S}$ represents the learning style from the ILS questionnaire, using a 3-item scale, and $n$ is the number of students. The function Sim compares its two parameters $L S_{\text {predicted }}$ and $L S_{I L S}$ and returns 1 if both are equal, 0.5 if one represents a balanced learning style and the other represents a preference for one of the two poles of the dimension, and 0 if they are opposite.

\subsection{Results}

Table 1 shows the results of the comparison between the proposed approach and the ILS questionnaire. The achieved results range from $73.33 \%$ to $79.33 \%$, demonstrating a high precision of the proposed approach for all dimensions of the FSLSM, and therefore, show that the proposed approach is suitable for identifying learning styles.

Table 1. Results of the comparison

\begin{tabular}{cccc}
\hline act/ref & sen/int & vis/ver & seq/glo \\
\hline $79.33 \%$ & $77.33 \%$ & $76.67 \%$ & $73.33 \%$ \\
\hline
\end{tabular}

\section{Discussion and related work}

The proposed approach is based on literature and applies a simple rule for calculating learning styles from indications gathered from the students' behaviour during an online course in an LMS. The underlying concept is quite similar to the concept of the ILS questionnaire, apart that in the automatic approach information from students' behaviour is used rather than asking students about their preferences.

Related works aim at identifying learning styles in specific learning systems rather than in LMSs in 
general. Furthermore, they focus on data-driven approaches, where data from students' behaviour and their learning styles were used in order to build a model for calculating learning styles. García et al. [10] applied Bayesian networks in order to detect learning styles for three dimensions of the FSLSM. In their final study, they achieved results of $58 \%$ for the active/ reflective dimension, $77 \%$ for the sensing/intuitive dimension, and $63 \%$ for the sequential/global dimension, using the same measure as proposed in Section 3.2. Another study was conducted by Cha et al. [12], investigating the use of Decision Trees and Hidden Markov Models. The results were promising, however, only data from the ILS questionnaire indicating a strong or moderate preference on a specific learning style dimension were considered and data indicating a balanced learning style were excluded. Therefore, further investigations towards a more accurate approach are necessary.

Another important issue for the automatic identification of learning styles is the considered number of patterns per learning style dimension. For the proposed approach, each learning style dimension consists of a relatively high number of patterns, compared to those of related works, such as the model introduced by García et al. [10] as well as one of our previous research work [13]. On one hand, a high number of patterns give more detailed information and, on the other hand, it helps to identify learning styles in LMSs in general since information about some patterns might not be available in all LMSs.

\section{Conclusions}

This paper introduced an automatic student modelling approach for identifying learning styles based on the FSLSM in LMSs. The proposed approach uses the behaviour of students during they are learning in order to gather hints about their learning styles. By applying a simple rule-based mechanism, learning styles are calculated based on the gathered indications. The evaluation of the approach demonstrated good results and showed that the approach is suitable for identifying learning styles with respect to the FSLSM.

Since the proposed approach is applicable for LMSs in general rather than for one specific system, it allows teachers to identifying their students' learning styles while they hold their courses in LMSs. The information about students' learning styles can be used for making students aware of their learning styles and providing them with courses/material that fit their learning styles.

Future work will deal with developing a concept for dynamic automatic student modelling, where data from students' behaviour will be used on the fly for modifying and updating the student model and therefore, allowing the system to immediately respond on students' needs.

\section{References}

[1] D.A. Kolb, Experiential Learning: Experience as the Source of Learning and Development, Prentice-Hall, Englewood Cliffs, New Jersey, 1984.

[2] P. Honey and A. Mumford, The Manual of Learning Styles. Peter Honey, Maidenhead, 1982.

[3] R.M. Felder and L.K. Silverman, Learning and teaching styles in engineering education, Engineering Education, 78,1988 , pp. 674-681. Preceded by a preface in 2002: http://www.ncsu.edu/felderpublic/Papers/LS-1988.pdf.

[4] N. Bajraktarevic, W. Hall, and P. Fullick, "Incorporating Learning Styles in Hypermedia Environment: Empirical Evaluation", Proceedings of the Workshop on Adaptive Hypermedia and Adaptive WebBased Systems, Nottingham, UK, 2003, pp. 41-52.

[5] S. Graf and Kinshuk, "Providing Adaptive Courses in Learning Management Systems with Respect to Learning Styles", Proceedings of the World Conference on E-Learning in Corporate, Government, Healthcare, and Higher Education (eLearn), AACE, 2007, pp. 2576-2583.

[6] P. Brusilovsky, Methods and techniques of adaptive hypermedia, User Modeling and User-Adapted Interaction, 6, 1996, pp. 87-129.

[7] Moodle, 2008. Available at http://www.moodle.org. Accessed 8 January, 2008.

[8] WebCT, 2008. Available at http://www.webct.com. Accessed 8 January, 2008.

[9] A.P. Rovai and K.T. Barnum, "On-line course effectiveness: an analysis of student interactions and perceptions of learning", Journal of Distance Education, 18 (1), 2003, pp. 57-73.

[10] P. García, A. Amandi, S. Schiaffino, and M. Campo, "Evaluating Bayesian Networks' Precision for Detecting Students' Learning Styles”, Computers \& Education, 49 (3), Elsevier, 2007, pp. 794-808.

[11] R.M. Felder and B.A. Soloman. Index of Learning Styles Questionnaire. Retrieved 6 January, 2008, from http://www.engr.ncsu.edu/learningstyles/ ilsweb.html

[12] H.J. Cha, Y.S. Kim, S.H. Park, T.B. Yoon, Y.M. Jung, and J.-H. Lee, "Learning Style Diagnosis Based on User Interface Behavior for the Customization of Learning Interfaces in an Intelligent Tutoring System", Proceedings of the 8th International Conference on Intelligent Tutoring Systems, Lecture Notes in Computer Science, Berlin, Heidelberg, Springer, Vol. 4053, 2006, pp. 513-524.

[13] S. Graf and Kinshuk, "An Approach for Detecting Learning Styles in Learning Management Systems", Proceedings of the International Conference on Advanced Learning Technologies (ICALT), Los Alamitos, CA, IEEE Computer Science, 2006, pp. 161163. 